\title{
Effect of in vitro and in vivo induction of polyethelene glycol-mediated osmotic stress on hybrid taro (Colocasia esculenta (L.) Schott)
}

\author{
Manas Ranjan Sahoo, Madhumita DasGupta and Archana Mukherjee \\ Regional Centre of Central Tuber Crops Research Institute, \\ Bhubaneswar - 751019, Orissa, India
}

\section{ABSTRACT}

Sahoo, M. R., M. DasGupta and A. Mukherjee. 2006. Effect of in vitro and in vivo induction of polyethelene glycol-mediated osmotic stress on hybrid taro (Colocasia esculenta (L.) Schott). Ann. Trop. Res. 28(2):1-11.

Taro hybrid TSL (Topi X Satasankha), along with its parental lines, was evaluated for stress tolerance under in vitro and in vivo polyethelene glycol (PEG) -mediated osmotic stress conditions. Aseptic cultures were raised in vitro under control and PEGmediated stress conditions. Stress index for survival and growth parameters like rooting ability in cultures and number of leaves produced per plantlet revealed the better response of hybrid lineTSL than its parental lines. The plantlets were hardened and transferred to pots for evaluation of morpho-physico-biochemical parameters under PEG-mediated osmotic stress conditions. Significant variations were observed in stress index for plant height, number of leaves, leaf area, \% relative water content (RWC), chlorophyll stability index (CSI), \% injury by dessication and yield. Decline in biochemical traits like protein content but increase in catalase and peroxidase activities was observed under the osmotic stress conditions. Less variation in morpho-physico-biochemical characters were recorded in TSL under stress conditions as compared to its parents. The hybrid lineTSL showed tolerance to osmotic stress with a minimum yield reduction. TSL can be used for the future breeding program for the development of drought tolerant lines and can become the source of favorable genes for drought tolerance in taro.

Keywords: hybrid taro, in vitro, in vivo, PEG-6000, osmotic stress, stress index

Correspondence: M. R. Sahoo Present Address: Regional Centre of Central Tuber Crops Research Institute, Dumduma Housing Board, Bhubaneswar - 751019, Orissa, India 6521-A Tel No +91 6742470528 Email: mrsahoo2004@indiatimes.com

DOI: $10.32945 /$ atr2821.2006 


\section{INTRODUCTION}

Taro (Colocasia esculenta (L.) Schott), a crop of immense commercial valuc, ranks fourtecnth among vegetable crops worldwide. India is said to be the secondary center of origin of taro enriched with tolerant traits to abiotic stresses. Taro grows in a wide range of agroecological conditions - swampy lands, uplands under irrigation and also as a rainfed crop (Wilson, 1984). Despite its wide adaptive nature, production of taro can be affected severely by stress.

Drought is a major constraint to successful agriculture in the arid and semi-arid tropics. It has been found that from 1939 to 1978 , over $40 \%$ of the total insurance indemnities for crop losses involved drought (Boyer, 1982). Hence, efforts are necessary to develop crop plants that can tolerate hostile environments. Some of the local cultivars of taro are found to have certain degree of tolerance to water deficit stress (Ravi and Chowdhury, 1997). However, cultivation under rainfed conditions can lead to severe yield loss $(90-100 \%)$ due to erratic monsoon. In order to augment the taro breeding program for genetic improvement, studies have already been initiated to screen 115 taro accessions for tolerance to drought (Sahoo et al., 2002). Polyethylene glycol 6000 ( the number signifying molecular mass) is most frequently used in plant water deficit studies (Ng and Ekanayake, 1997) to induce dehydration by decreasing the water potential of the nutrient solution (Jacomini et al., 1988). Hence, the present investigation was undertaken to evaluate the effects of in vitro and in vivo induction of polyethelene glycol-mediated osmotic stress on growth of hybrid-TSL and its parental lines- Topi and Satasankha.

\section{MATERIALS AND METHODS}

The experiment was conducted at the Regional Centre of Central Tuber Crops Research Institute, Bhubaneswar, India. The Centre is located at $20^{\circ}$ $15^{\prime} \mathrm{N}, 85^{\circ} 52^{\prime} \mathrm{E}$ at an altitude of $26 \mathrm{~m}$ above the mean sea level. It is about $60 \mathrm{~km}$ away from the Bay of Bengal. The climatic condition of the place is warm, moist with hot summer and comparatively mild winter. The taro accession Satasankha that shows moderate tolerance to drought was crossed with Topi that has good potential to develop the hybrid line TSL. Cormels 
obtained from the hybrid and its parents were used as materials for the present investigation.

\section{In vitro induction and evaluation for PEG-mediated stress}

In vitro cultures were raised by inoculating cormel tips of TSL, Topi and Satasankha in agar-solidified ( $8 \%$ ) Murasige and Skoog (MS) basal medium (Murashige and Skoog, 1962) supplemented with growth regulators [0.25 mg/l napthalene acetic acid (NAA), $0.5 \mathrm{mg} / \mathrm{l}$ benzyl adenine (BA) and $0.25 \mathrm{mg} / 1$ gibberellic acid $\left.\left(\mathrm{GA}_{3}\right)\right]$. The MS media with all growth regulators and $3 \%$ sucrose were used as control. In order to induce osmotic stress (-2 bars), the same media were supplemented with PEG-6000 (118g/1). The $\mathrm{pH}$ of the media was adjusted to 5.8 and then, the media were sterilized at $121^{\circ} \mathrm{C}$ for 20 minutes. Surface sterilization of taro was done following the procedure of Mukherjee et al. (1998). For each treatment, 20 replications were maintained at $25 \pm 1^{\circ} \mathrm{C}$ with 12 hours photoperiod and $30 \mu \mathrm{Em}^{-2} \mathrm{~s}^{-1}$ irradiance.

Survival rate of plantlets exposed to osmotic stress were calculated as the percentage of cultures with living plantlets. Other growth parameters such as rooting ability and number of leaves were recorded after 6 weeks of inoculation. In vitro raised plantlets were acclimatized, hardened in cups containing autoclaved soil and sand mixture $(1: 1 \mathrm{v} / \mathrm{v})$ and then, were subsequently established in pots under control and PEG-mediated stress conditions.

\section{In vivo induction and evaluation for PEG-mediated stress}

Potted cultures were laid out with in vitro plantlets to evaluate the effect of in vivo induction of PEG-mediated stress on TSL and its potential lines. The treated pots were irrigated with PEG-treated water $(118 \mathrm{~g} / \mathrm{l})$ while normal irrigation was given to control pots. Six replications were maintained for both conditions. The variations in morphological characters such as plant height, number of leaves per plant and leaf area were determined at 30,60 and 90 days after planting (DAP). The leaf area was calculated according to the linear measurement method of Biradar et al. (1978). Various physiological parameters like percentage relative water content (\%RWC), chlorophyll stability index (CSI) and percentage injury by desiccation ( $\%$ injury) were 
studied following the methodologies developed by Barrs and Weatherley (1962). Kolcyorcas (1958) and Sullivan (1971), respectively. Biochemical parameters like protein content of the PEG-treated and control plants were recorded using Bradford method as explained by Mahadevan and Sridhar (1996). The activity of the antioxidant enzymes, catalase and peroxidase, was assayed by following the methodologies of Aebi (1983) and Kar and Feirabend (1984) using a UV spectrophotometer at $240 \mathrm{~nm}$ and $470 \mathrm{~nm}$, respectively. The yicld was recorded at harvest. Stress index (S.I.) for each parameter was calculated following the methodology developed by Ng and Ekanayake (1997). Data were transformed by angular transformation and analyzed for significance using ANOVA.

\section{RESULTS AND DISCUSSION}

\section{In vitro induction and evaluation for PEG-mediated osmotic stress}

Significant variations in growth parameters were observed among the lines evaluated in vitro under PEG-mediated stress conditions. Application of PEG to differentiate crop responses to osmotic stress has been well documented ( $\mathrm{Ng}$ and Ekanayake, 1997). The stress index (SI) for survival was found to be lower in TSL $(22.16 \%)$ than Satasankha (30.29\%) and Topi $(37.12 \%)$ [F1g. 1]. SI for number of roots produced per plantlet was less in TSL $(40.69 \%)$ as compared to Satasankha $(45.20 \%)$ and Topi $(47.26 \%)$, whercas SI for the number of leaves produced per plantlet was recorded as low as $32.3 \%$ in TSL followed by $37.51 \%$ in Satasankha and $40.75 \%$ (Fig. 1). Similar results in survival of plantlets and growth retardations were noticed in cassava under PEG-mediated stress conditions (Ng and Ekanayake, 1997). Responses of cormel tips of hybrid and parental lines cultured on MS medium win' growth regulators along with PEG-6000 are presented in Fig.2A-C. Under in vitro stress conditions, multiple shoots formation was observed in the cormel tip cultures of hybrid taro (Fig. 2A). Satasankha responded well under stress conditions (Fig. 2B), whereas necrosis due to osmotic stress was observed in Topi (Fig. 2C). The present study indicates that the developed hybrid line TL 


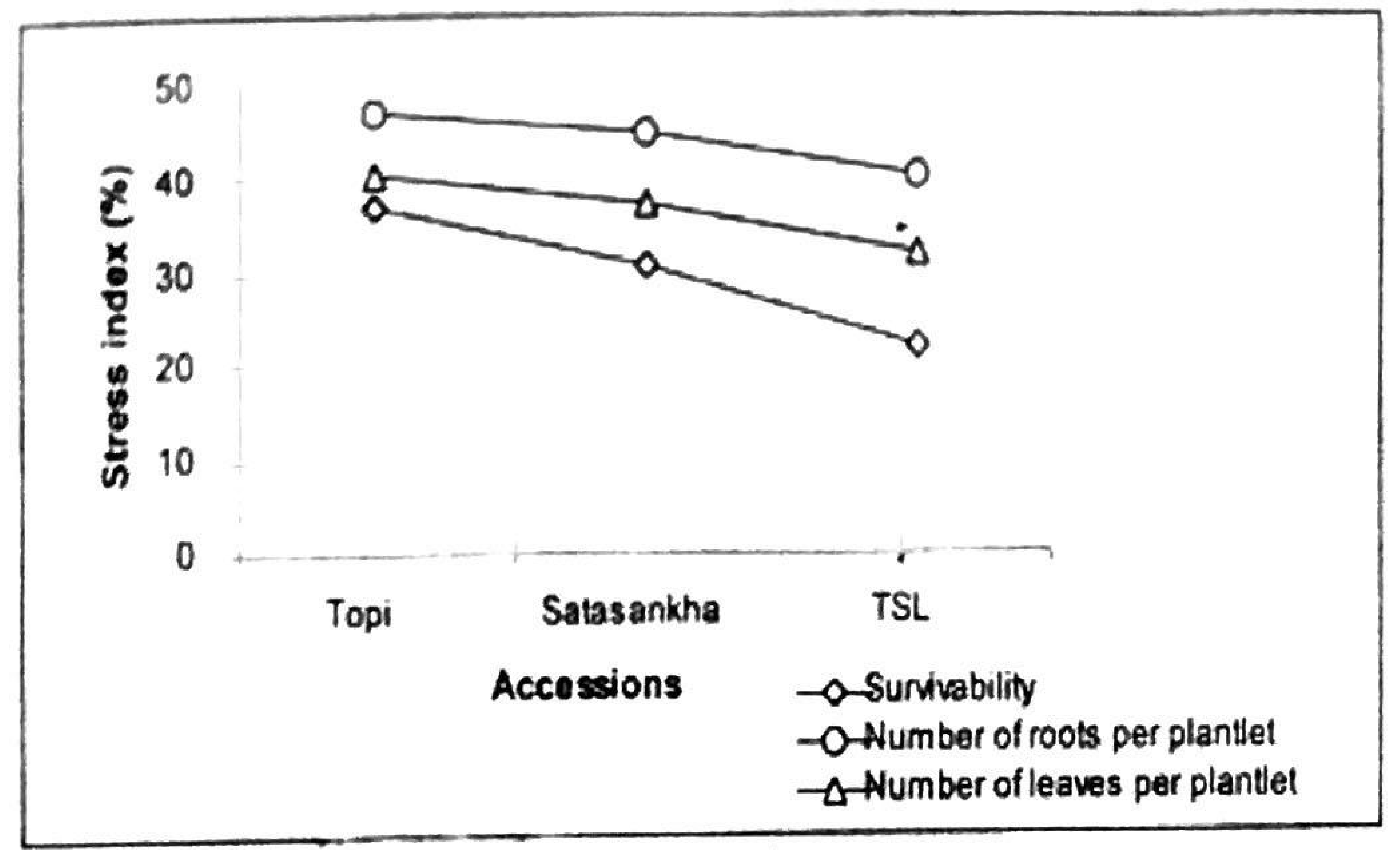

Figure 1. Effect of PEG on stress index for survival and growth parameters under in vitro conditions

showed more tolerance to osmotic stress with low SI for survival, rooting and leaf formation in vitro. In vitro raised plantlets were acclimatized, hardened in cups (Fig. 2D) containing sterilized soil and sand in equal proportion (1:1) and transferred subsequently to the pots for evaluation for stress tolerance (Fig. 2E).

In vivo induction and evaluation for PEG-mediated osmotic stress

Significant differences in retardations in growth parameters were observed among the lines evaluated under in vivo induced stress conditions as compared to the control. Plant height stress index (PHSI) was found to be significantly lower in TSL $(28.53 \%, 27.03 \%$ and $17.92 \%)$ than in Satasankha $(41.94 \%$, $31.56 \%$ and $29.71 \%$ ) and Topi $(44.51 \%, 37.71 \%$ and $34.80 \%)$ at 30,60 and 90 days after planting (DAP), respectively (Table 1). SI for number of leaves produced per plant was recorded to be $13.37 \%, 24.82 \%$ and $17.50 \%$ 

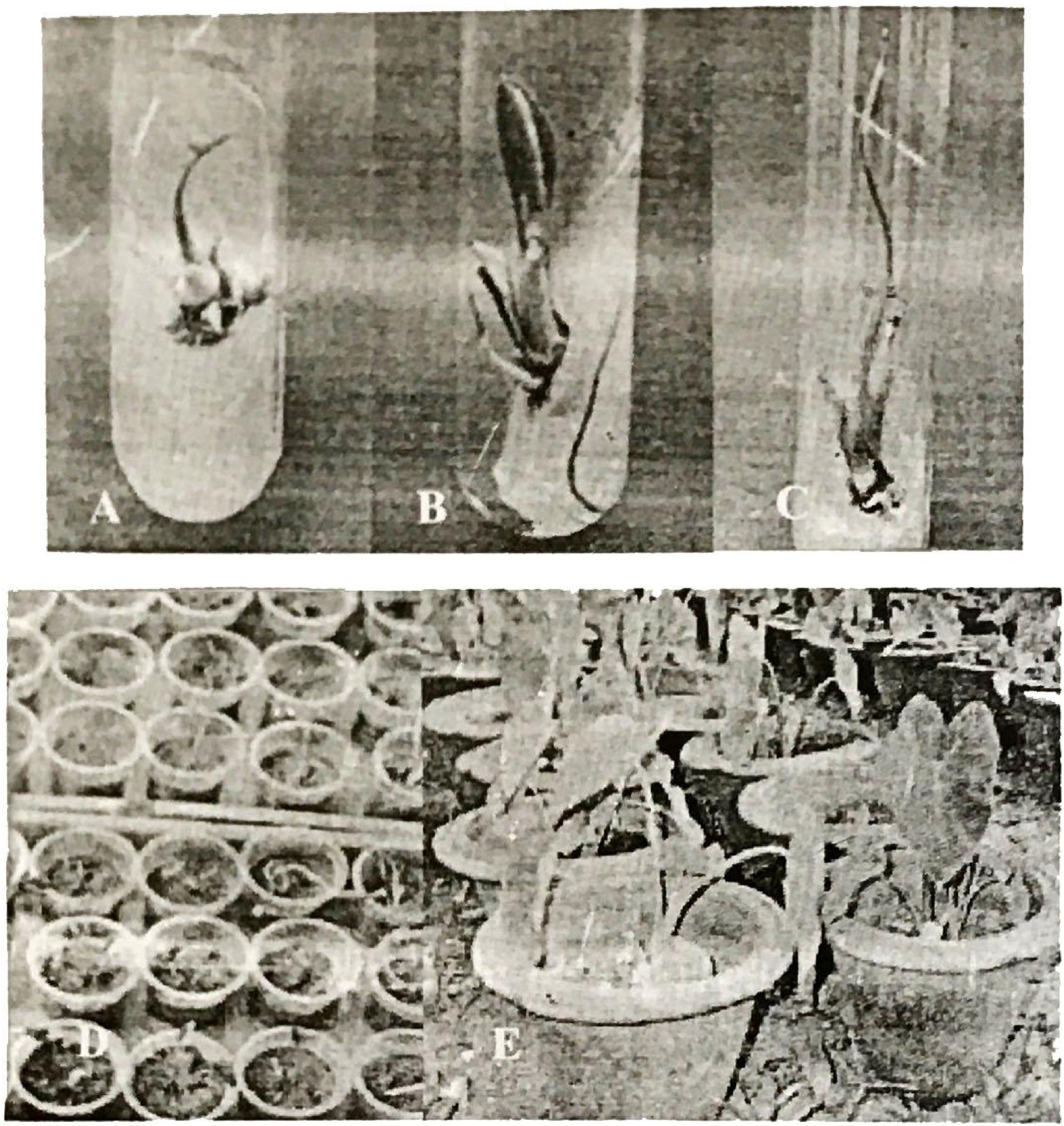

Figure 2. Multiple shoot produced from cormel tip in hybrid-TSL under PEG-mediated osmotic stress (A); cormel tip culture of Satasankha and Topi under stress (B and $\mathrm{C}$ ); acclimatization of in vitro plantlets; and evaluation for osmotic stress tolerance $(\mathrm{E})$ 
in TSL at 30,60, and 90 DAP, respectively. Corresponding values in Satasankha $(22.50 \%, 32.51 \%$ and $25.12 \%)$ and in Topi $(39.13 \%, 33.46 \%$ and $33.04 \%$ ) were higher. The stress index for leaf area was non significant at 30 DAP was significantly less in TSL at 60 and 90 DAP $(31.81 \%$ and $15.85 \%$ ). The leaf area stress index was recorded to be $56.12 \%$ and $15.94 \%$ in Satasankha and $47.29 \%$ and $38.08 \%$ in Topi at 60 and 80 DAP, respectively. This suggests decreased leaf area is an early adaptive response to water deficit and can be considered as a first line of defense against drought.

Significant variations in stress index for physiological parameters like percentage relative water content (\%RWC), chlorophyll stability index (CSI) and percentage injury by desiccation (\% injury) were observed among the lines. The SI for $\%$ RWC was lowest (1.56\%) in TSL followed by Satasankha $(1.84 \%)$ and Topi (3.77\%) (Table 2). Reduction in \%RWC was reported earlier in taro accessions growing under osmotic stress (Ravi and Chowdhury, 197). Similar trends among the three accessions were noted in stress indices for CSI and yield. Reduction in total chlorophyll content under water stress conditions was discussed earlier by Indira and Kabeerathumma (1998) in sweet potato and Ravi and Chowdhury (1997) in taro. A high CSI value reflects that the stress did not have much effect on chlorophyll content of plants which in turn helps the plants to withstand stress through better availability of chlorophyll.

Biochemical analysis revealed that the protein content ( $\mathrm{mg} / \mathrm{mg}$ of leaves) was decreased in PEG-treated plants (Fig. 3A). It has been found that production of reactive oxygen species (ROS) is common of water stress leading to degradation of lipids, proteins and DNA. However, results indicate increase in catalase and peroxidase activities ( $\mathrm{n} \mathrm{kat} / \mu \mathrm{g}$ protein) under stress conditions (Fig. 3B and C). The rate of increase in catalase and peroxidase activities under PEG-mediated osmotic stress conditions was higher in TSL than its parental lines which reflects its tolerance towards osmotic stress conditions. Probably higher level of these antioxidant enzymes in TSL play a vital role in scavenging of ROS and thereby minimizing the effect of stress.

A significant reduction in yield was observed among the lines under stress. Stress index for yield was recorded to be $24.20 \%$ in TSL, $44.45 \%$ in Satasankha and $52.31 \%$ in Topi (Table 2). The reduction in corm yield under water deficit stress was also attributed to reduction in crop growth rate, leaf 

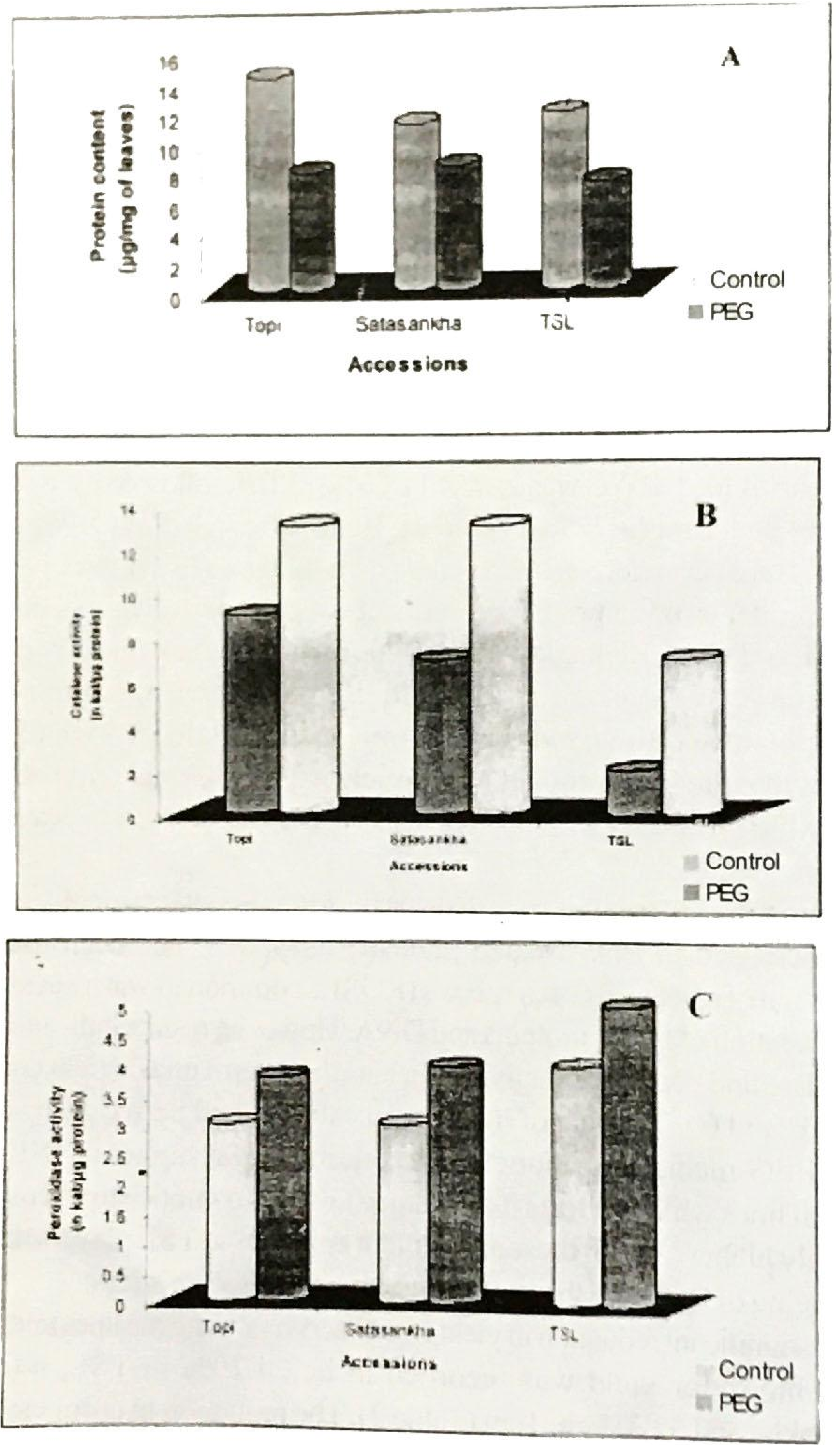

Figure 3. Effect of PEG on biochemical parameters in taro (A-C). 
Table 1. Effect of PEG on morphological parameters in taro

\begin{tabular}{lll} 
& Stress Index $(\%)$ & \\
\hline Plant Height & $\begin{array}{l}\text { Number } \\
\text { of leaves } \\
\text { plant }\end{array}$ & Leaf Area \\
& &
\end{tabular}

SI No. Accessions 30 DAP 60 DAP 90 DAP 30 DAP 60 DAP 90 DAP 30 DAP 60 DAP 90 DAP

\begin{tabular}{lclllllllll}
\hline 1 & Topi & 44.51 & 37.71 & 34.80 & 39.13 & 33.46 & 33.04 & 41.73 & 47.29 & 38.08 \\
2 & Satasankha & 41.94 & 31.56 & 29.71 & 22.50 & 32.51 & 25.12 & 40.66 & 56.12 & 15.94 \\
3 & TSL & 28.53 & 27.03 & 17.92 & 13.37 & 24.82 & 17.50 & 41.67 & 31.81 & 15.85 \\
& Mean & 38.3 & 32.1 & 27.5 & 25.0 & 30.3 & 25.2 & 41.4 & 45.0 & 23.3 \\
\hline \multicolumn{7}{c}{} \\
& SEm (0.05) 4.19 & 2.51 & 3.28 & 9.03 & 5.52 & 7.72 & NS & 3.13 & 3.48 \\
& C. D. at 5\% 12.63 & 7.55 & 9.88 & 27.21 & 16.64 & 23.26 & NS & 9.44 & 10.50 \\
\hline
\end{tabular}

Table 2. Effect of PEG on physiological parameters and yield in taro

\begin{tabular}{llllll}
\hline & & \multicolumn{4}{c}{ Stress Index (\%) } \\
\cline { 3 - 6 } SI No. & \multirow{2}{*}{ Accessions } & \%RWC & CSI & \% Injury & Yield \\
\hline 1 & Topi & 3.77 & 4.47 & 21.24 & 52.31 \\
2 & Satasankha & 1.84 & 4.39 & 11.36 & 4.45 \\
3 & TSL & 1.56 & 2.04 & 2.88 & 24.20 \\
& Mean & 2.4 & 3.6 & 11.8 & 40.3 \\
\hline & SEm(0.05) & 0.59 & 0.67 & 2.08 & 2.34 \\
& C. D. at 5\% & 1.77 & 2.01 & 6.28 & 7.05 \\
\hline
\end{tabular}


area and net assimilation rate (Ravi and Chowdhury, 1991).

\section{CONCLUSION}

From the results of the present investigation, it can be concluded that the hybrid line TSL responded well to in vitro induced osmotic stress conditions with high survivability and less growth retardations than its parental lines Topi and Satasankha. The hybrid line also showed tolerance with regard to morphophysico-biochemical parameters under in vivo $P E G$ mediated osmotic stress conditions. The hybrid TSL developed from moderately susceptible parent Satasankha and high yielding line of Topi can be included in the genetic improvement program to develop elite lines tolerant to osmotic stress.

\section{ACKNOWLEDGMENT}

The authors gratefully acknowledge the financial support of ICAR, NATP-CGP to carry out the present investigation. The authors are also thankful to the Head, Regional Centre of CTCRI, and Director of CTCRI for providing the necessary facilities to carry out the present study.

\section{REFERENCES}

AEBI, H. E. 1983. In: Methods of Enzymatic Analysis (H. U. Bergmeyer, J. Bergmeyer and M. Bl. Gra, eds.) 3rd Ed. Vol. 3. VCH, Weinheim, New York. 273-286 pp.

BARRS, H. C. and P. E. WEATHERLEY. 1962. A re-examination of the relative turgidity technique for estimating water deficits in leaves. Australian Biological Sciences. 15:413-428.

BIRADAR, R. S., T. VENKATESWARLU and N. HIRISH. 1978. Leaf area estimation in Colocasia. Root Crops. 4:51-53.

BOYER, J. S. 1982. Plant productivity and environment. Science. 218:443-448.

INDIRA, P. and S. KABEERATHUMMA. 1988. Physiological response of sweet potato under water stress: 1 . Effect of water stress during different phases of tuberization. Root Crops. 14:47-50. 
JAC OMINI, E..A. BERTANI and S. MAPELLI. 1988. Accumulation of PEG-6000 and its effects on water sontent and carbohydrate level in water stressed tomato plants. Canadian J Bot. 66:970-973.

KAR, M., and J. FEIERABEND. 1984. Changes in the activities of the enzymes involved in amino acid metabolism during the senescence of detached wheat leaves. Physiol. Plant 62:39-44.

KOLEYOREAS, S. A. 1958. A new method for determining drought resistance. Plant Physiol. 3:22.

MAHADEVAN, A. and R. SRIDHAR. 1996. Enzymes of infected plants and of parasites. In: Methods in Physiological Plant Pathology. Sivakami Publications, Chennai. $32 \mathrm{pp}$.

MUKHERJEE, A., B. K. DEBATA and S. K. NASKAR. 1998. Somatic embryogenesis and chromosomal ability of regenerants in sweet potato and taro. J. Scientific and Industrial Research. 57:709-715.

MURASHIGE, T. and F. SKOOG. 1962. A revised medium for rapid growth and bioassays with tobacco tissue culture. Physiol. Plant. 15:473-497.

NG S. Y. C. and I. J. EKANAYAKE. 1997. Screening cassava (Monihot esculenta Crantz) for polyehtelene glycol-mediated osmotic stress in vitro.J. Root Crops. 23(1):1518.

RAVI, V. and S. R. CHOWDHURY. 1997. Physiological response of taro to different soil moisture regimes. Root Crops. 17:129-133.

SAHOO, M. R., S. SAHU, A. MUKHERJEE, S. K. NASKAR and R. MISRA. 2002. In vitro screening of taro genetic resources for tolerance to biotic and abiotic stresses. In: Plant Resources Utilization (S. Sahoo, D. B. Ramesh, P. K. Panda and V. N. Misra, eds.) Allied Publishers, New delhi. 217-223 pp.

SULLIVAN, C. Y. Techniques for measuring plant drought stress. In: Drought Injury and resistance in crops (K. L. Larson and J. D. Eastin, eds.) Crop Science Society of America. Madison, Wisconsin.

WILSON, J. E. 1984. Cocoyam. In: The Physiology of tropical field crops (Goldworthy P. R. and N. M. Fisher, eds.) John Wiley and Sons Ltd. 589-604 pp. 